\title{
Uma Plataforma de Serviços para Mapas Conceituais
}

\author{
Wagner de A. Perin, Davidson Cury \\ Departamento de Informática - Universidade Federal do Espírito Santo (UFES) \\ Av. Fernando Ferrari, 514, 29075-910 - Vitória - ES, Brasil \\ Wagner.perindufes.br, dededinf.ufes.br
}

\begin{abstract}
In the educational context, interoperability of systems can be used to solve recurring problems in higher education in computer science and engineering courses, such as the insufficient theoretical and technological preparation and the difficulties encountered by teachers in engaging students with problems involving different types of knowledge. We propose a long-term project that, in addition to ensuring the integration of several developed computational solutions for many students, designs a complete platform for editing and management of concept maps with the specific purpose of supporting pedagogical practices based on constructivist theories of learning.
\end{abstract}

Resumo. No contexto educacional, a interoperabilidade dos sistemas pode ser usada para resolver problemas recorrentes no ensino superior, em cursos de ciência da computação e engenharia, como a insuficiente preparação teórica e tecnológica e as dificuldades encontradas pelos professores em envolver os alunos com problemas que lidam com diferentes tipos de conhecimento. Propomos um projeto de longo prazo que, além de garantir a integração de várias soluções computacionais desenvolvidas por estudantes, também projeta uma plataforma completa para edição e gestão de mapas conceituais com o objetivo específico de apoiar práticas pedagógicas baseadas em teorias construtivistas de aprendizagem.

\section{Introdução}

A interoperabilidade dos sistemas informatizados tem ampliado suas aplicações e transformado a rotina e as formas de trabalho de pessoas e organizações. Atualmente, qualquer pessoa pode criar, integrar e compartilhar informações, mídia e serviços em diferentes aplicações e reutilizar estas informações em diferentes serviços. Hoje em dia, muitas pessoas usam seus smartphones enquanto, por exemplo, realizam atividades físicas para compartilhar informações importante (tais como caminho, distância, duração e ritmo) em diferentes redes sociais. Estas mesmas informações podem ser exploradas por aplicativos que realizam, entre outras coisas, o controle de peso e a dieta dos usuários, ajudando-os no controle de obesidade. Sendo assim, uma pequena porção de informações podem ser enriquecidas pela enorme quantidade de serviços que as compartilham. Obviamente, gigantes da computação (tais como Google, Facebook e Amazon) possuem sistemas de recomendação quem se aproveitam de tais informações para oferecer produtos e serviços para o perfil do usuário.

No contexto educacional, a interoperabilidade dos sistemas pode ser usada para resolver certos problemas recorrentes no ensino superior em cursos de ciência da 
V Congresso Brasileiro de Informática na Educação (CBIE 2016)

Anais do XXVII Simpósio Brasileiro de Informática na Educação (SBIE 2016)

computação e engenharia. Entre esses problemas, citamos a preparação teórica e tecnológica insuficiente para atender as necessidades tanto da investigação científica mais avançada como do mercado de trabalho. De igual maneira, as dificuldades encontradas pelos professores em envolver os alunos em problemas que envolvam diferentes tipos de conhecimento já que os problemas geralmente apresentados aos estudantes são tão pequenos que as suas soluções desenvolvidas pela comunidade acadêmica desaparecem com o fim do semestre escolar.

Com isto em mente, nosso grupo de pesquisadores do Laboratório de Informática na Educação propõe um projeto de longo prazo que, além de garantir a integração de várias soluções computacionais desenvolvidas por estudantes de diferentes níveis (graduação, mestrado e doutorado), projeta uma plataforma completa para edição e gestão de mapas conceituais cujo propósito específico é dar apoio a práticas pedagógicas baseadas em teorias construtivistas de aprendizagem. O objetivo deste artigo é relatar como a adoção de aprendizagem baseada em problemas tem ajudado no desenvolvimento de uma percepção mais madura deste projeto. Assim, introduzimos o CMPaaS, ou seja, uma plataforma de serviços para mapas conceituais (Cury et al., 2014).

Neste artigo apresentaremos o contexto e a estrutura deste projeto, respondendo às seguintes questões norteadoras:

- Como ocorrem as integrações e porque são importantes para a educação em computação?

- Como o trabalho colaborativo e cooperativo beneficia a aprendizagem coletiva?

- Quais são os principais desafios e dificuldades enfrentadas pelo grupo?

- Como esses desafios estão sendo resolvidos?

- Que vantagens a arquitetura de sistema utilizada pode trazer para a educação em ciência e engenharia da computação?

O artigo está organizado em cinco seções, a saber: Seção 1, apresenta o contexto, a motivação, os objetivos e a organização deste artigo. A Seção 2 que mostra a natureza dos mapas e suas aplicações. A Seção 3 discute os métodos e a abordagem pedagógica do projeto. A Seção 4 apresenta os desafios e as tecnologias utilizadas para enfrentá-los. A Seção 5 apresenta o estado atual do projeto e, finalmente, a Seção 6 oferece algumas conclusões e os projetos futuros.

\section{Sobre a Natureza e Uso dos Mapas}

Um mapa conceitual é um conjunto de proposições lógicas que definem um contexto. Dependendo da abordagem que usamos, o mesmo texto pode gerar dois mapas de naturezas diferentes: um mapa contendo quaisquer proposições lógicas ou um mapa contendo proposições estritamente lógicas que definem as relações entre conceitos, de fato. Assumimos aqui que estes dois tipos de mapas cumprem os seguintes objetivos:

- Mapas conceituais para representar os processos da aprendizagem individual e coletiva, de crianças ou adultos, ou seja, mapas para fins educacionais. 
V Congresso Brasileiro de Informática na Educação (CBIE 2016)

Anais do XXVII Simpósio Brasileiro de Informática na Educação (SBIE 2016)

- Mapas conceituais para a engenharia do conhecimento. Aqui o mapa servirá como representação intermediária no processo de construção semiautomática de ontologias de domínio para uso em atividades de gestão do conhecimento, por exemplo. Este é o tipo que interessa à nossa pesquisa.

Em nosso mapa, os conceitos são substantivos e as ligações são estruturas verbais. Cada tripla (conceito, ligação, conceito) forma uma unidade semântica, a menor unidade de informação presente em um mapa conceitual, o qual denominamos de proposição. As proposições constituem a característica básica dos mapas conceituais, aquilo que os distingue de outras representações semelhantes [Dutra et al, 2007]. A Figura 1 mostra um exemplo de um mapa conceitual de um aluno de mestrado que explora o tema "Desenvolvimento e Aprendizagem em Piaget".

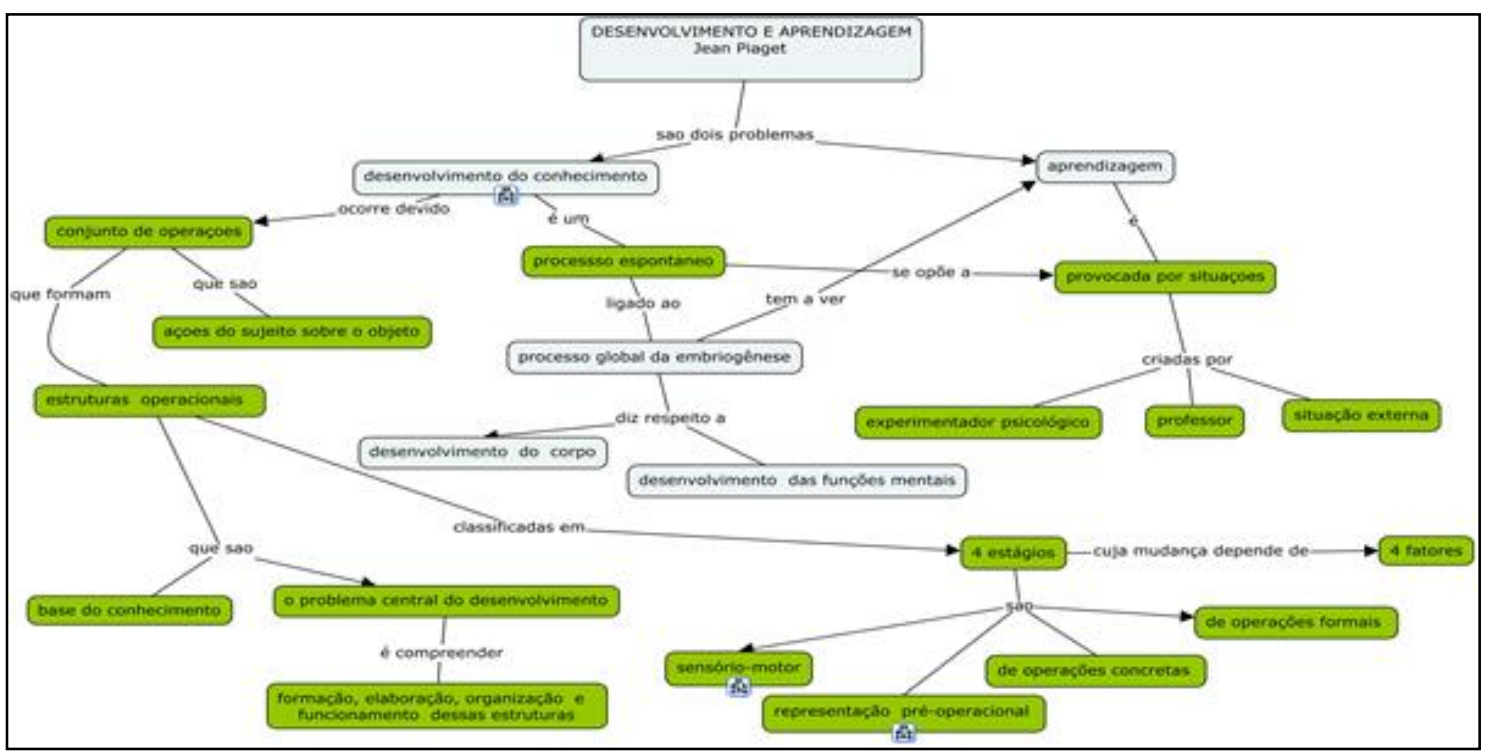

Figura 1. Um Mapa Conceitual.

Por nossas experiências, podemos afirmar, com um alto grau de certeza, que há inúmeros contextos onde os mapas conceituais podem servir como uma ferramenta muito útil. Em geral, começamos a partir de um texto para obter um mapa equivalente. No entanto, também podemos fazer o oposto, isto é, ir de um mapa para gerar um texto. É claro que os mapas não substituem os textos, mas são um bom começo para a sua construção. A partir de um único mapa, vários textos podem ser gerados, dependendo da visão e interpretação de cada leitor.

Estamos interessados em explorar e melhorar as muitas características positivas que observamos em mapas conceituais tanto nos processos de ensino-aprendizagem regular quanto em aplicações que envolvam a engenharia do conhecimento e memória organizacional. Destacamos também o uso dos mapas na educação infantil, quando eles são adequados para crianças com dificuldades na construção de frases. Porque lúdicos, eles incentivam e facilitam-lhes expressar suas ideias.

\subsection{Os Mapas além da Educação}

A engenharia do conhecimento tem usado ontologias para a gestão do conhecimento. A gestão do conhecimento é funcionalmente envolvida na geração, armazenamento e 
V Congresso Brasileiro de Informática na Educação (CBIE 2016)

Anais do XXVII Simpósio Brasileiro de Informática na Educação (SBIE 2016)

disseminação do conhecimento dentro de qualquer corporação. Ela engloba várias atividades, que vão desde técnicas de recursos humanos até técnicas relacionadas com os sistemas de informação.

Dos mapas podemos obter as ontologias. As ontologias, por outro lado, para serem manipuladas por computador, são representadas em lógica descritiva. Elas têm desempenhado um papel importante na gestão do conhecimento no que diz respeito à construção de interfaces homem-máquina intuitivas e adaptativas, recuperação inteligente de informação, entre outras atividades. Ontologias também têm sido utilizadas para capturar o conhecimento sobre algum domínio de interesse.

Os mapas conceituais são usados no contexto da gestão do conhecimento, principalmente porque são considerados um instrumento eficaz para compartilhar o conhecimento de um determinado domínio. Além disso, eles são bons para ajudar na construção de comunicação consensual entre especialistas de domínio e engenheiros de conhecimento [Cañas \& Novak, 2008].

\section{O Método de Trabalho}

O trabalho proposto consiste na definição de um método e a utilização de uma tecnologia adequada ao método, ambos apresentados a seguir.

\subsection{O Contexto}

Nosso trabalho é baseado na ideia estratégica de criar um laboratório para a formação contínua de pessoas nos planos teóricos, tecnológicos e conceituais sobre desenvolvimento e gestão de projeto. Para este fim, o projeto deve necessariamente ser de longa duração, não só capaz de manter um grupo ao longo da sua vida acadêmica, mas também capaz de incorporar novas gerações de professores e alunos. Suas dimensões devem se estender além dos limites físicos, aproveitando as oportunidades criadas pela tecnologia. Guiada pela mistura de diferentes teorias cognitivas de aprendizagem, a sua organicidade deve ser predisposta a favor da construção cooperativa e colaborativa do conhecimento do grupo de trabalho.

Os grupos de trabalho são heterogêneos, reunindo estudantes de graduação, mestrado e doutorado. Os professores também têm diferentes habilidades, são de diferentes universidades e até mesmo de diferentes países. Para esse grupo, seu currículo deve ser uma alternativa para o clássico, necessária para explorar as deficiências e potencialidades individuais. Para tal currículo, a metodologia terá de quebrar a clássica educação disciplinar. Deve também facilitar a construção adaptativa e individual do conhecimento, devendo fornecer leituras de acordo com a necessidade do projeto. Além disso, deve promover a sinergia do grupo.

Os grupos se reestruturam todo tempo, de acordo com as necessidades do momento. Assim, num dado momento estudantes mais avançados ajudam os outros. Em outro momento, problemas individuais são tratados por todo o grupo e ainda em outro, todos os estudantes são expostos a um problema genérico que pode gerar um extenso brainstorming. Ao longo do tempo, as soluções antigas são discutidas e novas propostas tecnológicas são incorporadas, gerando novas versões. Da mesma forma, o grupo deve ter em mente, o tempo todo, que os conceitos são dinâmicos e podem mudar. Assim, 
V Congresso Brasileiro de Informática na Educação (CBIE 2016)

Anais do XXVII Simpósio Brasileiro de Informática na Educação (SBIE 2016)

uma visão crítica profunda e constante é incentivada, sugerindo a possibilidade de ressignificações.

\subsection{A Abordagem Pedagógica}

As dificuldades encontradas no processo de ensino e aprendizagem das disciplinas iniciais de programação estão relacionadas com o desenvolvimento de várias habilidades necessárias para programar computadores que, em geral, não são consideradas antes dos estudantes se inscreverem na Universidade. Entre essas habilidades estão:

- Domínio de uma metodologia específica para a resolução de problemas, focada na construção de artefatos que atendam a um conjunto de requisitos;

- Domínio de uma metodologia para a avaliação da qualidade dos programas produzidos;

- Capacidade de resolver problemas de forma cooperativa;

- Uso da sintaxe, da semântica e pragmática de uma linguagem de programação;

- Facilidade de uso de diferentes ferramentas computacionais de apoio ao desenvolvimento e avaliação de programas.

Temos trabalhado com arquiteturas pedagógicas que são capazes de suportar uma certa disciplina. No contexto do CMPaaS, a intenção é promover um conjunto de disciplinas, não previamente programadas, que surgirá de acordo com a necessidade do projeto [Tavares et al, 2012]. Sabemos também que a pesquisa em informática na educação tem mostrado que as tecnologias digitais têm desempenhado um papel importante no desenvolvimento das habilidades acima mencionadas [Nitzke, 2006]. No entanto, acreditamos que a tecnologia sem o apoio de uma teoria adequada de aprendizagem resolve pouco. Portanto, investimos em aspectos da teoria construtivista de Piaget que são capazes de mudar qualitativamente este cenário.

Nosso projeto se desenvolve em torno de um núcleo que garante uma longa duração. Esse projeto tem como objetivo gerar um produto de software livre, fácil de usar e disponível para a comunidade em geral. Além disso, ele tem as características de um projeto de pesquisa que visa a construção de conhecimento coletivo, caracterizandose como um projeto de ensino-aprendizagem. Os mapas conceituais podem garantir isso. Assim, escolher o CMPaaS como projeto que tornará a abordagem viável.

No desenvolvimento deste projeto adotamos um método de trabalho que estende as características de aprendizagem baseada em problemas. Ao contrário do ensino tradicional baseado no ensino, nossa metodologia muda o foco para a aprendizagem, tornando o estudante o principal agente no processo. Uma vez organizado em grupos de trabalho, os estudantes passam a identificar o problema, investigar, discutir, interpretar e produzir soluções possíveis ou recomendações. Como se pode ver, a cooperação e a colaboração definem o método de trabalho. Nele, os professores tornam-se mediadores na construção do conhecimento.

A Figura 2 permite compreender como ocorre a integração entre os diversos projetos desenvolvidos pela comunidade acadêmica envolvida no projeto e, também, como as fronteiras impostas pela pesquisa são rompidas, permitindo o desenvolvido e integração de serviços produzidos pela comunidade externa. 
V Congresso Brasileiro de Informática na Educação (CBIE 2016)

Anais do XXVII Simpósio Brasileiro de Informática na Educação (SBIE 2016)

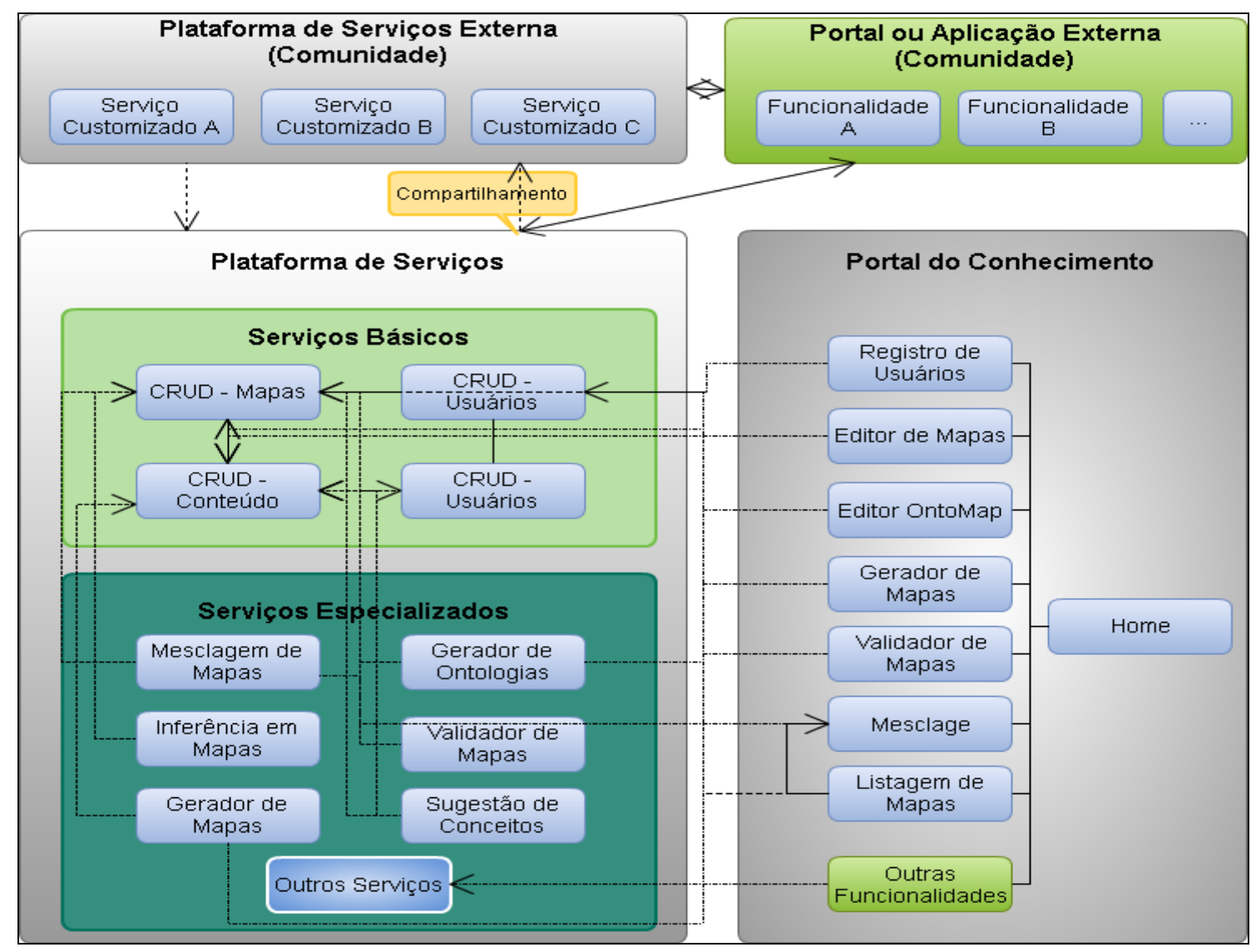

Figura 2. Arquitetura conceitual do Projeto CMPaaS.

A parte inferior da Figura 2 mostra a Plataforma de Serviços e o Portal do Conhecimento. A Plataforma de Serviço consiste de uma coleção de WebServices que realizam tarefas fundamentais do projeto, ou seja, consiste dos processos operacionais para criação, atualização, leitura e exclusão de mapas conceituais e dados de usuários, além de outros Serviços Especializados. Já o portal do conhecimento, caracteriza-se como uma interface de interação entre os usuários finais e os serviços oferecidos na plataforma. Cada projetista de serviço é responsável por produzir uma interface que favoreça a interação com o serviço produzido na plataforma, o conjunto de serviços desenvolvidos internamente foi batizado como Portal do Conhecimento. Já a parte superior da figura demonstra que os serviços internos da Plataforma podem ser explorados por uma plataforma desenvolvida pela comunidade para estender suas funções, ou consumidas por um Portal também customizado por eles. Isso é vantajoso para a comunidade, pois serviços básicos podem ser reaproveitados podendo a comunidade se concentrar nos serviços finais que são de seu real interesse. Ou seja, num dado instante, um Portal desenvolvido por qualquer outra instituição pode estar oferecendo serviços da plataforma do projeto CMPaaS e estendo suas funções com serviços próprios, que são de seu exclusivo interesse.

\section{Desafios e as Tecnologias Exploradas}

A principal característica que exploramos no projeto CMPaaS é a sua capacidade de realizar a integração do grupo de trabalho. O trabalho cooperativo e colaborativo é essencial para o sucesso do projeto como um todo. No entanto, qual a forma de 
V Congresso Brasileiro de Informática na Educação (CBIE 2016)

Anais do XXVII Simpósio Brasileiro de Informática na Educação (SBIE 2016)

assegurar essa integração? E como incentivar a troca de informações, experiências e resultados da equipe? Como manter o conhecimento histórico produzido pelo grupo ao longo do tempo? Estas são algumas das perguntas que nos têm perturbado durante o processo de desenvolvimento do projeto.

\subsection{Os Desafios}

A gestão de um projeto com as características do CMPaaS traz consigo uma série de preocupações. O grupo envolvido no projeto é formado por pessoas de diferentes características, como já mencionamos. No entanto, existem outros desafios a serem considerados. Vamos listar alguns deles para apresentar as ferramentas tecnológicas que estão nos ajudando a supera-los.

1. Segmentação do grupo de trabalho: Atualmente o grupo é composto de quatro equipes, uma delas desenvolve os serviços básicos da plataforma enquanto as outras trabalham em serviços especializados. No entanto, a integração deve permitir o compartilhamento de informações importantes, o que significa dizer que a qualquer momento uma pessoa, ou um grupo, pode desenvolver e integrar novos serviços à plataforma. Garantir que esta integração se torne possível é um desafio e requer uma boa definição de normas e regras para a comunicação entre os serviços.

2. Situações de conflito: Ocorrência de conflitos internos é comum em grupos de trabalho diversificados. Às vezes, os membros de um subgrupo ou subgrupos distintos podem entrar em desacordo sobre como resolver um problema específico. Como lidar com essas situações? Como garantir que a solução tomada para resolver um conflito está disponível para todos os membros, a fim de evitar a sua repetição? Estes e outros conflitos relacionados ao gerenciamento de questões também é um desafio existente durante o andamento deste projeto.

3. Histórico da manutenção do desenvolvimento do Projeto: Para manter um registro histórico do desenvolvimento do projeto é essencial para acompanhar a sua evolução. Acreditamos que, no futuro, as pessoas vão se interessar e querer conhecer a história, versões e serviços que foram desenvolvidos, bem como publicações e erros / soluções que marcaram o desenvolvimento de pesquisas relacionadas a cada serviço oferecido pela plataforma. Assim, escolhemos e definimos uma maneira de registrar a evolução deste projeto.

4. Mediação e orientação: Visto que os estudantes estão em processo de aprendizagem e não detêm conhecimentos que permitam tomada de decisões importantes, a mediação/orientação do estudante precisa de atenção especial onde a comunicação e feedback das ações tomadas pelos membros precisam de agilidade para não comprometer uma condução harmônica do projeto. Diante de tal complexidade, optamos por um modelo de gestão distribuída, onde os pesquisadores responsáveis pelas soluções computacionais também procuram mediar e orientar o grupo, usando as ferramentas escolhidas para o projeto.

5. Normas de compartilhamento para o desenvolvimento do projeto: Padrões de compartilhamento são essenciais para a ocorrência de integração, não apenas das soluções desenvolvidas, mas também para promover a comunicação e, como consequência, o trabalho cooperativo. $\mathrm{O}$ que, como e onde organizar os padrões adotados também tem sido outro desafio neste projeto. 
V Congresso Brasileiro de Informática na Educação (CBIE 2016)

Anais do XXVII Simpósio Brasileiro de Informática na Educação (SBIE 2016)

6. A comunicação entre os membros do grupo: Visto que operamos com prazos flexíveis para realização de tarefas, para um trabalho de equipe eficaz, tem sido necessário utilizar soluções tecnológicas que facilitam a comunicação entre os membros do grupo, de modo a não comprometer o cronograma do projeto. Em situações de dúvida, os membros do grupo podem usar as ferramentas de comunicação para procurar a ajuda de membros da equipe.

7. Documentação das soluções propostas: No desenvolvimento do CMPaaS foi necessário padronizar a forma como as soluções desenvolvidas seriam documentadas para facilitar a compreensão por futuros integrantes da equipe ou até mesmo pelas partes externas interessadas.

8. Administração de progresso do projeto: Neste projeto, estamos adotando ferramentas de gerenciamento que ajudam a identificar as tarefas que compõem o caminho crítico do projeto para controle e gestão do cronograma do projeto.

A seção seguinte apresenta as soluções tecnológicas que nos ajudam a superar estes desafios.

\subsection{Tecnologias versus Desafios}

$\mathrm{Na}$ escolha por soluções tecnológicas para enfrentar os desafios apresentados anteriormente, foi dado prioridade para ferramentas gratuitas, já que o projeto não conta com aporte financeiro para seu desenvolvimento, a maioria dos estudantes não recebe qualquer ajuda financeira para desenvolver este projeto, trabalhando exclusivamente em prol do desejo de aprender. Em muitos casos, eles dividem o tempo dedicado ao projeto com suas atividades profissionais fora da academia que lhes garante algum recurso financeiro. A seguir, mostraremos, sumariamente, as soluções tecnológicas utilizadas no projeto, destacando suas potencialidades para ajudar a enfrentar os desafios.

Para a cooperação e colaboração, usamos o PBworks ${ }^{1}$, onde o agendamento de reuniões e registro de atas, o compartilhamento / discussão dos padrões adotados no projeto, a mediação de conflitos e gestão dos grupos de trabalho, são mantidos. Para um quadro de anotações, usamos o Trello $^{2}$, um quadro branco com ferramentas para a visualização do progresso das tarefas e consulta dos responsáveis por cada uma delas. Para o controle de versões e manutenção do histórico da evolução do projeto, adotamos o $G I T^{3}$, um sistema de controle de revisão distribuída com ênfase na velocidade, integridade de dados e suporte para fluxos de trabalho distribuídos, não-lineares [Chacon, 2014]. Para mensagens instantâneas, usamos o WhatsApp ${ }^{4}$. Para documentar as soluções computacionais desenvolvidas pelo grupo, a fim de torna-las compreensíveis para os desenvolvedores futuros, internos ou externos ao projeto, e assegurar a reutilização de soluções existentes e sua integração efetiva com os serviços que vierem estender os recursos existentes, escolhemos o Swagger ${ }^{5}$ [Haupt et al, 2014].

\footnotetext{
${ }^{1}$ PBWorks: Uma ferramenta Wiki de fácil manejo e uso, mais informações em http://www.pbworks.com

2 Trello: Uma ferramenta para gerencia de tarefas de projetos, mais informações em https://trello.com

${ }^{3}$ GIT: Sistema de controle de versão distribuído, mais informações em https://git-scm.com

${ }^{4}$ WhatsApp: Aplicação para mensagens instantâneas, mais informações em https://www.whatsapp.com

${ }^{5}$ Swagger: Framework para descrição de Serviços RESTful, disponível em http://swagger.io
} 
V Congresso Brasileiro de Informática na Educação (CBIE 2016)

Anais do XXVII Simpósio Brasileiro de Informática na Educação (SBIE 2016)

Este conjunto de soluções tecnológicas têm auxiliado no processo de gestão do desenvolvimento da plataforma, combatendo os desafios apresentados na seção anterior. A seção seguinte apresenta o estado atual da plataforma.

\section{O CMPaaS Hoje}

A Figura 3 apresenta a página inicial do Portal do Conhecimento, a principal interface de interação com os serviços oferecidos pela plataforma CMPaaS. Atualmente, o Portal do Conhecimento conta com os seguintes serviços da plataforma:

- Construção, edição e formatação de mapas conceituais: os usuários podem criar mapas conceituais online em diferentes dispositivos.

- Registro, perfil e autenticação do usuário: permite o registro e fornece acesso a serviços autenticados.

- Persistência e repositório de mapas: permite aos usuários salvar e recuperar seus mapas.

- Versionamento de mapas conceituais: permite ao usuário criar diferentes versões do mesmo mapa.

- Integração com o CmapTools: permite aos usuários importar/exportar mapas conceituais editados no CmapTools.

- Mecanismo de inferência: permite ao usuário fazer perguntas sobre o conteúdo do mapa, em linguagem natural [Perin et al., 2012].

- Um editor de ontologias: permite gerar ontologias rasas a partir de um mapa [Pinotte et al., 2015].

- Geração automática de mapas a partir de textos [Pinotte et al., 2015].

- Mesclagem de mapas conceituais [Vassoler et al., 2014].

- Validação estrutural de mapas conceituais [Assis et al., 2014].

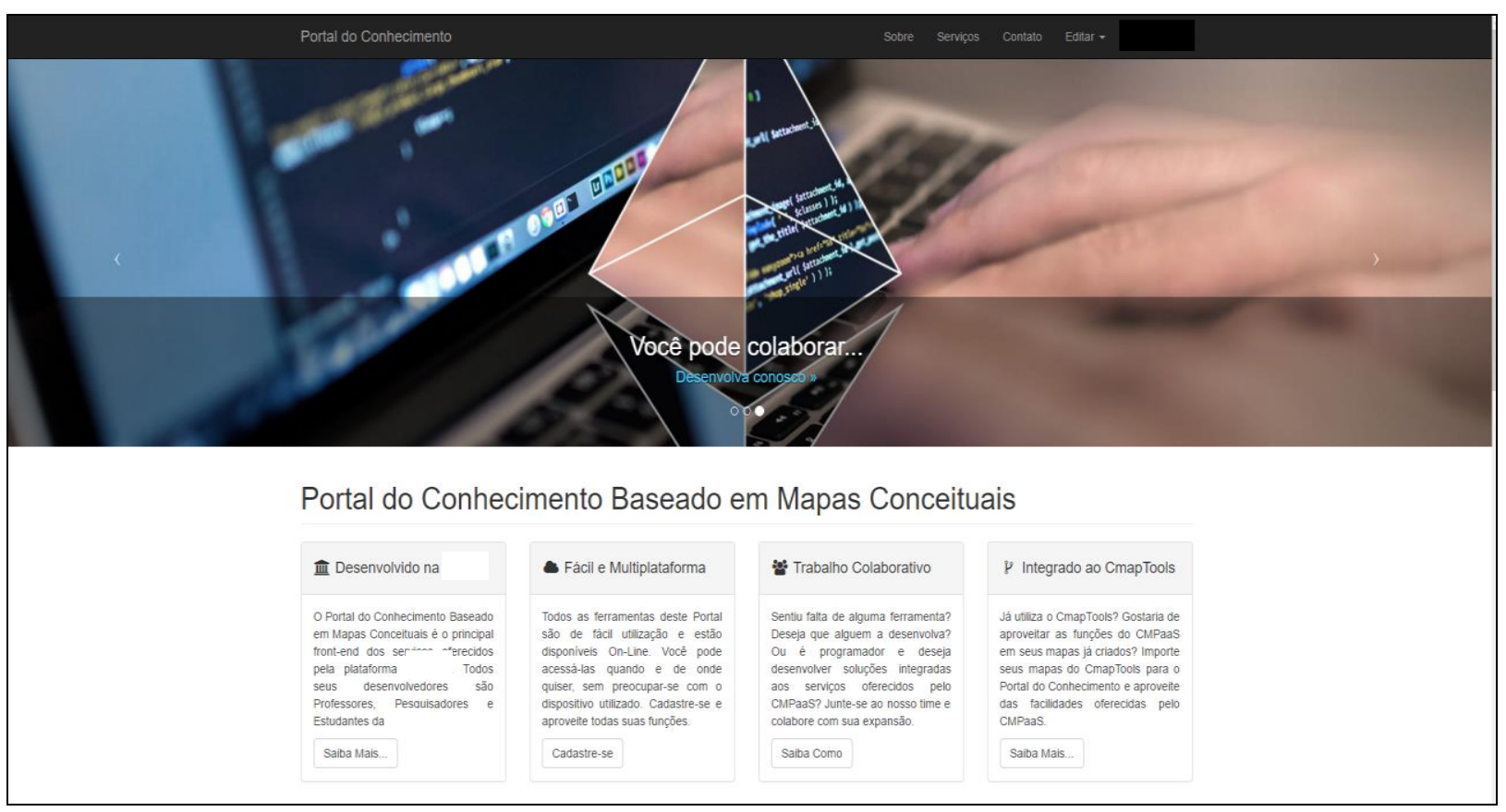

Figura 3. Página Inicial do Portal do Conhecimento. 
V Congresso Brasileiro de Informática na Educação (CBIE 2016)

Anais do XXVII Simpósio Brasileiro de Informática na Educação (SBIE 2016)

\section{Conclusões e Trabalhos Futuros}

Apresentamos neste artigo alguns resultados parciais de um projeto de longo prazo orientado a processos de ensino-aprendizagem onde princípios construtivistas que enfatizam, entre outras coisas, a atividade cooperativa como um meio para alcançar formas mais elevadas de consciência e de conhecimento, permitindo o estabelecimento de uma estratégia para construção de um laboratório de formação contínua de alunos em teorias, tecnologias e planejamento para gerenciar projetos. Apontamos os desafios e escolhemos diferentes tecnologias capazes de resolvê-los. Apresentamos a plataforma de serviços web CMPaaS que estará disponibiliza gratuitamente diferentes ferramentas para uso de mapas conceituais. Além disso, os desenvolvedores interessados no assunto, CMPaaS irá permitir-lhes adicionar novas ferramentas que ainda não figurem na sua lista.

\section{Referências}

Assis, Daniel V.; Perin, Wagner de A.; Vassoler, Geraldo A.; Cury, Davidson. VMAP Caracterização de uma Abordagem para Verificação Sintática de Mapas Conceituais. In: Sixth International Conference On Concep Mapping, Santos - SP, 2014.

Cañas, A. J., J. D. Novak, Facilitating the Adoption of Concept Mapping Using CmapTools to Enhance Meaningful Learning, In: Knowledge Cartography: Software Tools and Mapping Techniques, ed. A. L. P. Okada et al., Springer Verlag, 2008.

Chacon, S. Pro Git (2nd ed.). New York, NY. December 24, 2014: Apress. pp. 29-30. ISBN 978-1484200773.

Cury, Davidson; Perin, Wagner de A.; Santos Jr., Paulo S. CMPaaS - A Plataform of Services for Construction and Handling of Concept Maps. In: Sixth International Conference On Concep Mapping, Santos - SP, 2014.

Dutra, Í. M. ; Piccinini, C. A. ; Souza, L. A. S. de ; Costa, B. G. ; Estrázula, M. B. P. Desenho de uma Metodologia de Intervenção a Distância para Apropriação e Uso dos Mapas Conceituais em Atividades Educacionais. RENOTE. Revista Novas Tecnologias na Educação, v. 5, p. 1-10, 2007.

Haupt, F.; Karastoyanova, D.; Leymann, F.; Schroth, B. A Model-Driven Approach for REST Compliant Services. ICWS 2014. 2014 IEEE International Conference on Web Services: 129-136. doi:10.1109/ICWS.2014.30. ISBN 978-1-4799-5054-6.

Nitzke, J. The Insertion of the Information and Communication Technologies (ICT) in the practice of engineering. In the XXXI Brazilian Congress of Engineering Teaching - COBENGE. Sep 2006, Rio de Janeiro.

Perin, Wagner de A.; Cury, Davidson. . Construindo Mapas Conceituais utilizando a abordagem iMap. In: Congreso Internacional de Informática Educativa, 2012, Santiago. Anais do Evento, 2012. v. 1. p. 108-113.

Pinotte, Guilherme N., Cury. Davidson, Zouaq, Amal. OntoMap: De Mapas Conceituais a Ontologias OWL. Nuevas Ideas en Informática Educativa. In: Congreso Internacional de Informática Educativa, 2015, Santiago. Anais do Evento, 2015. V.1. p. $172-180$.

Tavares, O, Menezes, C., Nevado, R., "Pedagogical architectures to support the process of teaching and learning of computer programming", FIE 2012, Seatle, USA.

Vassoler, Geraldo A.; Perin, Wagner de A.; Cury, Davidson. MergeMaps - A Computational Tool for Merging of Concept Maps. In: Sixth International Conference On Concep Mapping, Santos - SP, 2014. 